\title{
Diagnóstico De Aprobación De La Certificación Internacional ESOL Examinations En La Modalidad Extensión Del Centro De Idiomas - ESPOCH
}

\author{
Enrique Jesús Guambo Yerovi \\ Magister en Lingüística Aplicada al Aprendizaje del Idioma Inglés \\ Docente de la Escuela Superior Politécnica de Chimborazo, \\ Centro de Idiomas, Ecuador \\ Marcelo Eduardo Allauca Peñafiel \\ Magister en Interconectividad de Redes \\ Técnico Docente de la Escuela Superior Politécnica de Chimborazo, \\ Centro de Idiomas, Ecuador
}

Doi: 10.19044/esj.2018.v14n2p81 URL:http://dx.doi.org/10.19044/esj.2018.v14n2p81

\begin{abstract}
This paper focuses on diagnosing and determining the number of students who obtained the International Certification of ESOL Examinations of Cambridge University, which was formed in the Extension Modality of the Language Center. For this reason, this study was carried out based on the academic follow up of those students who passed or failed the 8 levels of English. This was done with a total of 680 hours, planned through the dimensions of a new curriculum, designed within the project titled "Strengthening of the Extension Modality in the Language Center at the Higher Polytechnic School of Chimborazo" based on an academic text of Cambridge University. The results correspond to a total of 122 students of 4 promotions that were evaluated by skills and according to parameters established by the staff of the University of Cambridge ESOL Examinations. This, however, enabled us to ascertain the number of certifications recognized by the Common European Framework, obtained by the students in levels A2, $\mathrm{B} 1$, and $\mathrm{B} 2$.
\end{abstract}

Keywords: Diagnostics, International Certification, Cambridge ESOL

\section{Resumen}

El objetivo de la presente investigación fue diagnosticar y determinar el número de estudiantes que obtuvieron la Certificación Internacional de ESOL Examinations de Cambridge que se formaron en la Modalidad de Extensión del Centro de Idiomas. Por este motivo, se realizó el seguimiento 
académico de los estudiantes quienes aprueban y reprueban los ocho niveles de inglés con un total de 680 horas planificadas a través de las dimensiones de un nuevo currículo diseñado dentro del proyecto "Fortalecimiento de la Modalidad Extensión en el Centro de Idiomas de la Escuela Superior Politécnica de Chimborazo", basadas en un texto académico de Cambridge. Los resultados corresponden a un total de 122 estudiantes de cuatro promociones que fueron evaluados por destrezas y según parámetros establecidos por el personal de la Universidad de Cambridge ESOL Examinations y que nos permitió conocer el número de certificaciones reconocidas por el Marco Común Europeo, obtenidas por los estudiantes en los niveles A2, B1, y B2.

Palabras-Claves: Diagnóstico, Certificación Internacional, ESOL Cambridge.

\section{Introducción}

En Ecuador, tras obtener una puntuación de 46.90 en la tercera edición del índice anual de nivel de manejo de inglés "Education First" (EF) se ubica en el puesto 48 de los 60 países no angloparlantes analizados. El mismo estudio reveló que los jóvenes de 18 y 19 años, al culminar el bachillerato, tienen uno de los niveles más bajos de Sudamérica y esa diferencia no desaparece en la Universidad. La realidad ocasiona que los institutos de aprendizaje de inglés se vuelven una alternativa (El Comercio, 2014).

América Latina es la más débil de todas las regiones, con un nivel medio de inglés que apenas sobrepasa el límite más bajo del índice de nivel de inglés EPI, medido por Education First. De acuerdo al ranking del 2011, el Ecuador se encuentra en el puesto 37 de entre 42 países que toma en cuenta el estudio catalogado como un nivel muy bajo.

En marzo pasado, la SENESCYT presentó los resultados del nivel de inglés en el país. Según María Isabel Cruz, subsecretaria de esa institución, el promedio es de 13/20 y los profesores tienen un nivel inicial de inglés (Ecuador Inmediato, 2012).

"El promedio general en la materia de inglés en los estudiantes de décimo año de educación cásica como los estudiantes de tercer nivel de bachillerato aquí en el Ecuador es de 13/20. Nuestros profesores de inglés tienen un nivel inicial y así están enseñando clases”, señaló.

Para Susana Cabeza de Vaca, directora ejecutiva de la Comisión Fullbright, en el país hay una brecha que separa el aprendizaje del idioma entre los colegios ubicados en las grandes ciudades y aquellos de provincia.

Según dijo, el Gobierno está realizando un esfuerzo para capacitar a los profesores, pero se debería ofrecer al menos dos horas diarias de inglés a los alumnos, recogió Radio Quito. 
El Marco Común Europeo de Referencia, establece una serie de niveles para todas las lenguas a partir de los cuales se favorece la comparación u homologación de los distintos títulos emitidos por las entidades certificadas (Kaftandjieva Felianka, 2004).

Recopilando información acerca de los distintos niveles propuestos por otras entidades y autores, se observa que el número más adecuado de niveles para el aprendizaje, enseñanza y evaluación de las lenguas es seis, que no coinciden con los niveles clásicos de básico, intermedio y avanzado, al estar situados por encima o por debajo de ellos, respectivamente. Con base en esto, se elabora una distinción inicial en tres bloques amplios, cada bloque se ramifica en otros dos niveles más restrictivos.

Bloque A: Usuario básico.

- $\quad$ Nivel A1: Acceso.

- $\quad$ Nivel A2: Plataforma.

Bloque B: Usuario independiente.

- $\quad$ Nivel B1: Umbral.

- $\quad$ Nivel B2: Avanzado.

El desarrollo posterior del Marco Común Europeo de Referencia permite la simplificación del esquema anterior al siguiente, que facilita en gran medida la comprensibilidad a los usuarios de la información, presentando una tabla de escala global para los seis niveles (Council of Europe, 2001).

Nivel A1: Se adquiere cuando el estudiante es capaz de comprender y utilizar expresiones cotidianas de uso muy frecuente así como frases sencillas destinadas a satisfacer necesidades de tipo inmediato; cuando puede presentarse a sí mismo y a otros, pedir y dar información personal básica sobre su domicilio, sus pertenencias y las personas que conoce y cuando puede relacionarse de forma elemental siempre que su interlocutor hable despacio y con claridad y esté dispuesto a cooperar.

Nivel A2: Se adquiere cuando el estudiante es capaz de comprender frases y expresiones de uso frecuente relacionadas con áreas de experiencia que le son especialmente relevantes (información básica sobre sí mismo y su familia, compras, lugares de interés, ocupaciones, etc.); cuando sabe comunicarse a la hora de llevar a cabo tareas simples y cotidianas que no requieran más que intercambios sencillos y directos de información sobre cuestiones que le son conocidas o habituales y cuando sabe describir en términos sencillos aspectos de su pasado y su entorno así como cuestiones relacionadas con sus necesidades inmediatas.

Nivel B1: Se adquiere cuando el estudiante es capaz de comprender los puntos principales de textos claros y en lengua estándar si tratan sobre cuestiones que le son conocidas, ya sea en situaciones de trabajo, de estudio o de ocio; cuando sabe desenvolverse en la mayor parte de las situaciones que pueden surgir durante un viaje por zonas donde se utiliza la lengua; cuando es 
capaz de producir textos sencillos y coherentes sobre temas que le son familiares o en los que tiene un interés personal y cuando puede describir experiencias, acontecimientos, deseos y aspiraciones, así como justificar brevemente sus opiniones o explicar sus planes.

Nivel B2: Se adquiere cuando el estudiante es capaz de entender las ideas principales de textos complejos que traten de temas tanto concretos como abstractos, incluso si son de carácter técnico siempre que estén dentro de su campo de especialización; cuando puede relacionarse con hablantes nativos con un grado suficiente de fluidez y naturalidad de modo que la comunicación se realice sin esfuerzo por parte de ninguno de los interlocutores y cuando puede producir textos claros y detallados sobre temas diversos así como defender un punto de vista sobre temas generales indicando los pros y los contras de las distintas opciones.

Gracias al nuevo micro currículo que se ha implementado a través del proyecto de fortalecimiento de la modalidad extensión, que se llevó a cabo en un lapso de tres años. Los estudiantes tuvieron la posibilidad de prepararse y ser evaluados durante 8 niveles de inglés con un total de 720 horas presenciales con el fin de que rindan el examen de certificación internacional con el objetivo de que al menos el $60 \%$ obtenga certificaciones B1 y/o B2.

\section{Metodología}

La investigación es de tipo descriptiva y tuvo como población cuatro promociones de estudiante que se prepararon a través de una guía y formación de niveles de inglés y como parte del proyecto del Fortalecimiento de la Modalidad Extensión del Centro de Idiomas de la Escuela Superior Politécnica de Chimborazo, a través de un nuevo micro currículo. Los estudiantes se inscribieron $y$ se sometieron a la evaluación para la obtención de certificaciones internacionales en los niveles A2, B1 y B2 reconocidas por el Marco Común Europeo (Council of Europe, 2001).

La muestra representa 122 estudiantes quienes fueron evaluados en diferentes fechas. Los datos obtenidos representan las evaluaciones de destrezas bajo parámetros establecidos por el personal externo al Centro de idiomas y que representan a la Universidad de Cambridge ESOL Examinations. Los datos obtenidos fueron formalizados individualmente a cada estudiante, así como también una copia para la Dirección del Centro de Idiomas. La información obtenida fue analizada, tabulada e interpretada a través de tablas y gráficos a través de estadística descriptiva.

Se registra en la presente investigación el seguimiento de la última promoción previo a rendir el examen de certificación internacional en donde se realiza un seguimiento de los estudiantes que aprobaron y reprobaron los diferentes niveles. 
Para la obtención de la información, en primera instancia se realizó un registro de inscripción con el derecho de pago para rendir el examen. Los evaluadores confirmaron el listado de estudiantes, para posteriormente notificar su aceptación de forma individual. El personal evaluador.

Para el estudio de los resultados se consideró dos escenarios. El primero consideró los resultados por promociones previstas en las fechas que rindieron la evaluación. El segundo escenario recogió la información del número total de certificaciones por nivel. El personal evaluador de la Universidad de Cambridge ESOL Examinations solicitó al Centro de Idiomas requerimientos mínimos para la aplicación del examen de evaluación previa a la certificación en las que consta un laboratorio de cómputo con adecuaciones de mobiliario individual para cada estudiante, computadoras con características tecnológicas mínimas y conexión de internet. Cumplidos los requerimientos por parte del Centro de Idiomas, los evaluadores aplicaron la evaluación a los estudiantes de 4 promociones en diferentes fechas. Los resultados fueron entregados de forma individual con copia a la Dirección del Centro de Idiomas. Finalmente, con la información obtenida, se procedió al procesamiento de la información a través de estadística descriptiva por medio de la tabulación, análisis interpretación con tablas y gráficos estadísticos.

\section{Resultados}

\section{Resultados Nivel I}

Tabla 1. Total de estudiantes aprobados y reprobados del Nivel I, Abril 2015 - Julio 2015

\begin{tabular}{cc}
\hline ESTADO & NÚMERO \\
\hline Estudiantes aprobados & 874 \\
Estudiantes reprobados & 206 \\
Total & 1080 \\
\hline
\end{tabular}

Fuente: Actas de calificaciones.

Realizado por: Allauca Marcelo, 2015.

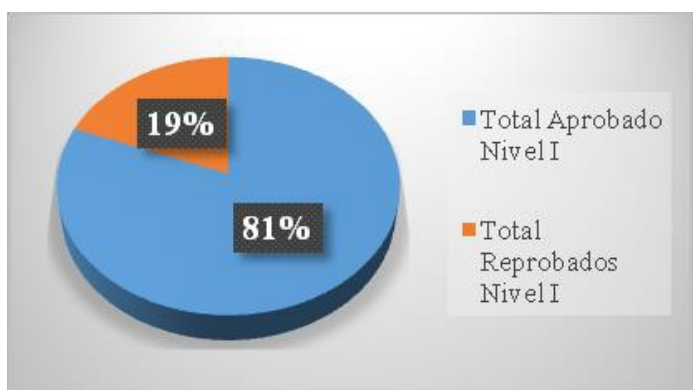

Gráfico 1. Total de estudiantes aprobados vs total de estudiantes reprobados del Nivel I, abril 2013 - julio 2015. 


\section{Análisis e interpretación}

Del $100 \%$ de los estudiantes que cursaron el primer nivel de inglés en los diferentes periodos académicos, el $81 \%$ aprobaron, mientras que el 19\% reprobaron. Es decir, la mayoría de los estudiantes aprobaron el nivel de inglés I en los diferentes periodos académicos.

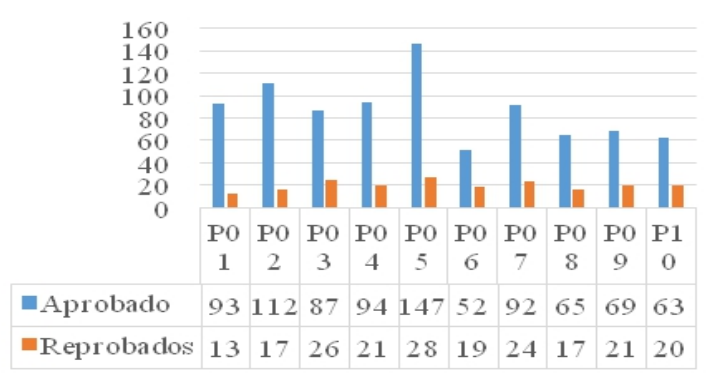

Gráfico 2.Total de estudiantes aprobados vs total de estudiantes reprobados de los diferentes paralelos del Nivel I, abril 2015 - julio 2015.

\section{Análisis e interpretación}

El Nivel I se dictó en 10 periodos académicos desde abril 2013 a julio 2015. En el periodo P05 se registró el mayor número de estudiantes aprobados; mientras que en el periodo P06 se registró el menor número de estudiantes aprobados. En el periodo P05 se registró el mayor número de estudiantes reprobados; mientras que en el periodo P01 se registró el menor número de estudiantes reprobados.

\section{Resultados Nivel II}

Tabla 2.Total de estudiantes aprobados y reprobados del nivel II, abril 2015 - julio 2015

\begin{tabular}{|c|c|}
\hline Estado & Número \\
\hline Estudiantes aprobados & 599 \\
\hline Estudiantes reprobados & 124 \\
\hline Total & 723 \\
\hline
\end{tabular}

Fuente: Actas de calificaciones.

Realizado por: Allauca Marcelo, 2015.

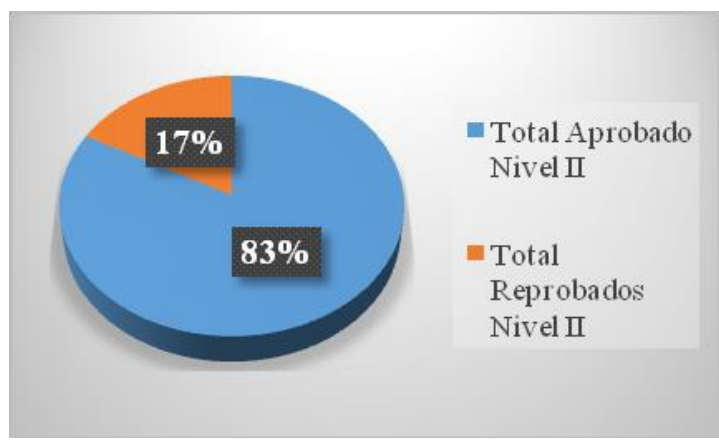

Gráfico 3. Total de estudiantes aprobados vs total de estudiantes reprobados del Nivel II, junio 2013 - julio 2015. 


\section{Análisis e interpretación}

Del 100\% de los estudiantes que cursaron el segundo nivel de inglés en los diferentes periodos académicos, el $83 \%$ aprobaron, mientras que el $17 \%$ reprobaron. Es decir, la mayoría de los estudiantes aprobaron el nivel de inglés II en los diferentes periodos académicos.

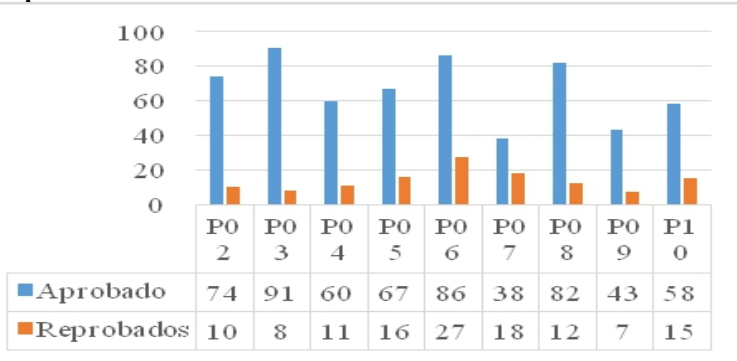

Gráfico 4. Total de estudiantes aprobados vs total de estudiantes reprobados del Nivel II, junio 2013 - julio 2015.

\section{Análisis e interpretación}

El nivel II se dictó en 9 periodos académicos desde junio 2013 a julio 2015. En el periodo P03 se registró el mayor número de estudiantes aprobados; mientras que en el periodo P07 se registró el menor número de estudiantes aprobados. En el periodo P06 se registró el mayor número de estudiantes reprobados; mientras que en el periodo P09 se registró el menor número de estudiantes reprobados.

\section{Resultados Nivel III}

Tabla 3.Total de estudiantes aprobados y reprobados del nivel III, septiembre 2013 - julio 2015

\begin{tabular}{cc}
\hline Estado & Número \\
\hline Estudiantes aprobados & 410 \\
Estudiantes reprobados & 75 \\
Total & 485 \\
\hline
\end{tabular}

Fuente: Actas de calificaciones.

Realizado por: Allauca Marcelo, 2015.

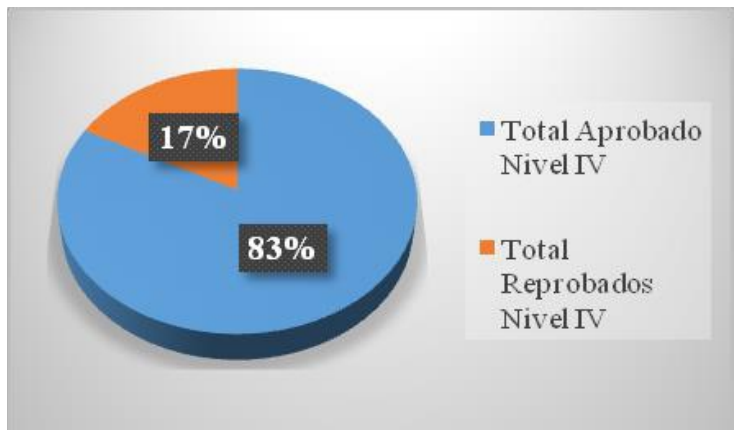

Gráfico 5. Total de estudiantes aprobados vs total de estudiantes reprobados del Nivel III, septiembre 2013 - julio 2015. 


\section{Análisis e interpretación}

Del $100 \%$ de los estudiantes que cursaron el tercer nivel de inglés en los diferentes periodos académicos, el $83 \%$ aprobaron, mientras que el $17 \%$ reprobaron. Es decir, la mayoría de los estudiantes aprobaron el nivel de inglés III en los diferentes periodos académicos.

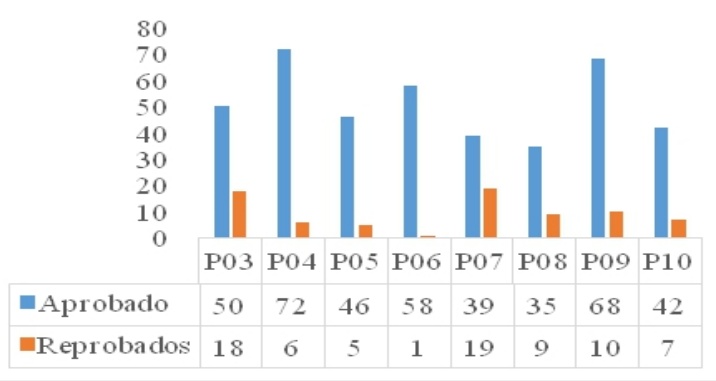

Gráfico 6. Total de estudiantes aprobados vs total de estudiantes reprobados del Nivel III, septiembre 2013 - julio 2015.

\section{Análisis e interpretación}

El nivel III se dictó en 8 periodos académicos desde septiembre 2013 a julio 2015. En el periodo P04 se registró el mayor número de estudiantes aprobados; mientras que en el periodo P08 se registró el menor número de estudiantes aprobados. En el periodo P07 se registró el mayor número de estudiantes reprobados; mientras que en el periodo P05 se registró el menor número de estudiantes reprobados.

\section{Resultados Nivel IV}

Tabla 4. Total de estudiantes aprobados y reprobados del nivel IV, noviembre 2013 -julio 2015

\begin{tabular}{cc}
\hline Estado & Número \\
\hline Estudiantes aprobados & 303 \\
Estudiantes reprobados & 63 \\
Total & 366 \\
\hline
\end{tabular}

Fuente: Actas de calificaciones.

Realizado por: Allauca Marcelo, 2015.

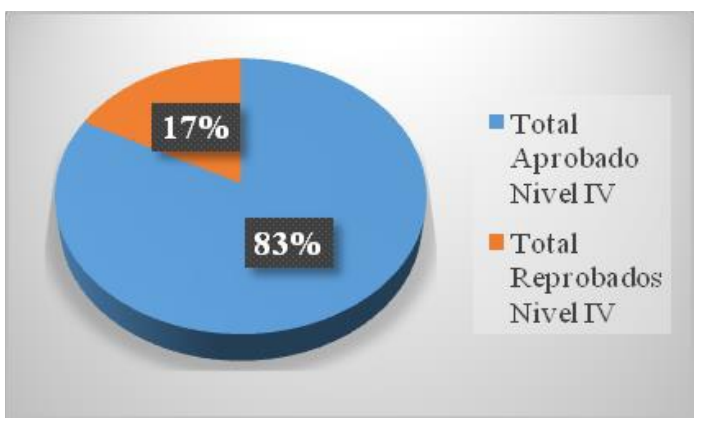

Gráfico 7. Total de estudiantes aprobados vs total de estudiantes reprobados del Nivel IV, noviembre 2013 - julio 2015. 


\section{Análisis e interpretación}

Del $100 \%$ de los estudiantes que cursaron el cuarto nivel de inglés en los diferentes periodos académicos, el $83 \%$ aprobaron, mientras que el $17 \%$ reprobaron. Es decir, la mayoría de los estudiantes aprobaron el nivel de inglés IV en los diferentes periodos académicos.

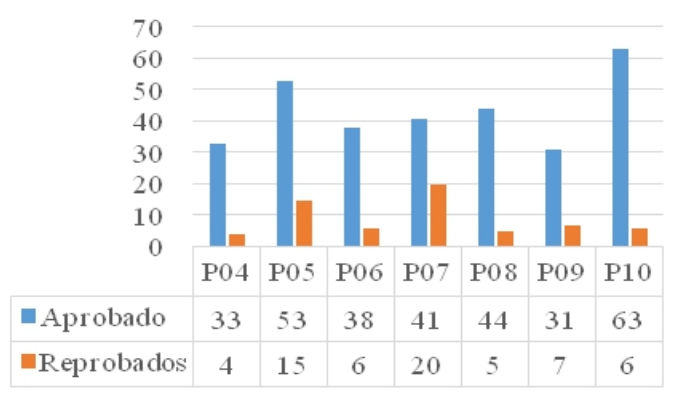

Gráfico 8. Total de estudiantes aprobados vs total de estudiantes reprobados del Nivel IV, noviembre 2013 - julio 2015.

\section{Análisis e interpretación}

El nivel IV se dictó en 7 periodos académicos desde noviembre 2013 a julio 2015. En el periodo P10 se registró el mayor número de estudiantes aprobados; mientras que en el periodo P04 se registró el menor número de estudiantes aprobados. En el periodo P07 se registró el mayor número de estudiantes reprobados; mientras que en el periodo P04 se registró el menor número de estudiantes reprobados.

\section{Resultados Nivel V}

Tabla 5. Total de estudiantes aprobados y reprobados del Nivel V, abril 2013 - julio 2015.

\begin{tabular}{|c|c|}
\hline Estado & Número \\
\hline Estudiantes aprobados & 195 \\
\hline Estudiantes reprobados & 13 \\
\hline Total & 208 \\
\hline
\end{tabular}

Fuente: Actas de calificaciones.

Realizado por: Allauca Marcelo, 2015.

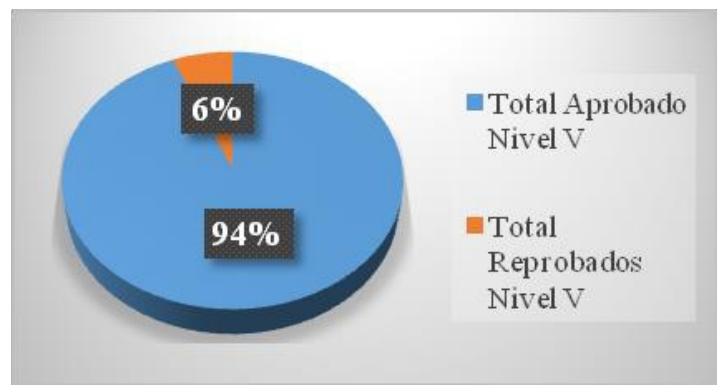

Gráfico 9. Total de estudiantes aprobados vs total de estudiantes reprobados del Nivel V, marzo 2013 - julio 2015. 


\section{Análisis e interpretación}

Del 100\% de los estudiantes que cursaron el quinto nivel de inglés en los diferentes periodos académicos, el 94\% aprobaron, mientras que el 6\% reprobaron. Es decir, la mayoría de los estudiantes aprobaron el nivel de inglés $\mathrm{V}$ en los diferentes periodos académicos.

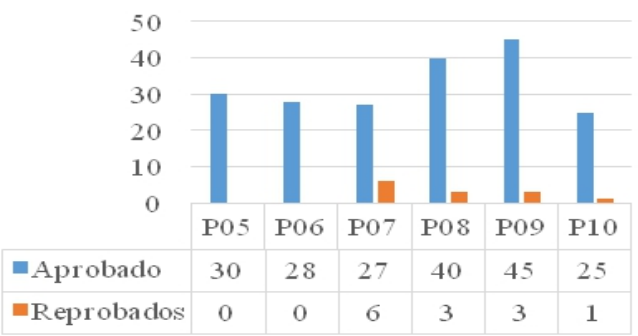

Gráfico 10. Total de estudiantes aprobados vs total de estudiantes reprobados del Nivel V, marzo 2013 - julio 2015.

\section{Análisis e interpretación}

El nivel V se dictó en 6 periodos académicos desde marzo 2013 a julio 2015. En el periodo P09 se registró el mayor número de estudiantes aprobados; mientras que en el periodo P07 se registró el menor número de estudiantes aprobados. En el periodo P07 se registró el mayor número de estudiantes reprobados; mientras que en el periodo P09 se registró el menor número de estudiantes reprobados. En los periodos P05 y P06 no existió estudiantes reprobados.

\section{Resultados Nivel VI}

Tabla 6.Total de estudiantes aprobados y reprobados del Nivel VI, junio 2013 - julio 2015.

\begin{tabular}{cc}
\hline Estado & Número \\
\hline Estudiantes aprobados & 180 \\
Estudiantes reprobados & 22 \\
Total & 202 \\
\hline
\end{tabular}

Fuente: Actas de calificaciones.

Realizado por: Allauca Marcelo, 2015.

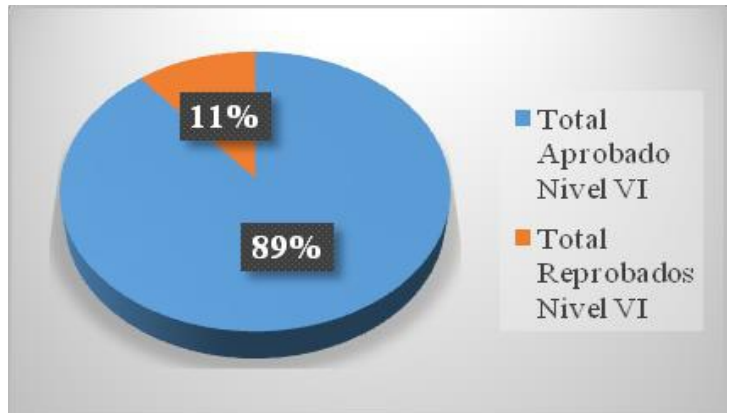

Gráfico 11. Total de estudiantes aprobados vs total de estudiantes reprobados del Nivel VI, junio 2013 - julio 2015. 


\section{Análisis e interpretación}

Del $100 \%$ de los estudiantes que cursaron el sexto nivel de inglés en los diferentes periodos académicos, el $89 \%$ aprobaron, mientras que el $11 \%$ reprobaron. Es decir, la mayoría de los estudiantes aprobado el nivel de inglés VI en los diferentes periodos académicos.

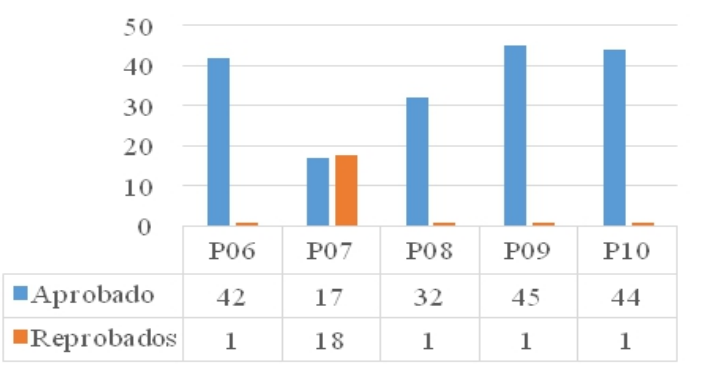

Gráfico 12. Total de estudiantes aprobados vs total de estudiantes reprobados del Nivel VI, junio 2013 - julio 2015.

\section{Análisis e interpretación}

El Nivel VI se dictó en 5 periodos académicos desde junio 2013 a julio 2015. En el periodo P10 se registró el mayor número de estudiantes aprobados; mientras que en el periodo P07 se registró el menor número de estudiantes aprobados. En el periodo P07 se registró el mayor número de estudiantes reprobados; mientras que en los periodos P08, P09 y P10 se registraron el menor número de estudiantes reprobados. En el periodo P07 se registró que el número de estudiantes reprobados fue mayor al número de aprobados.

\section{Resultados Nivel VII}

Tabla 7. Total de estudiantes aprobados y reprobados del nivel VII, septiembre 2013 - julio 2015.

\begin{tabular}{cc}
\hline Estado & Número \\
\hline Estudiantes aprobados & 120 \\
Estudiantes reprobados & 1 \\
Total & 121 \\
\hline
\end{tabular}

Fuente: Actas de calificaciones.

Realizado por: Allauca Marcelo, 2015.

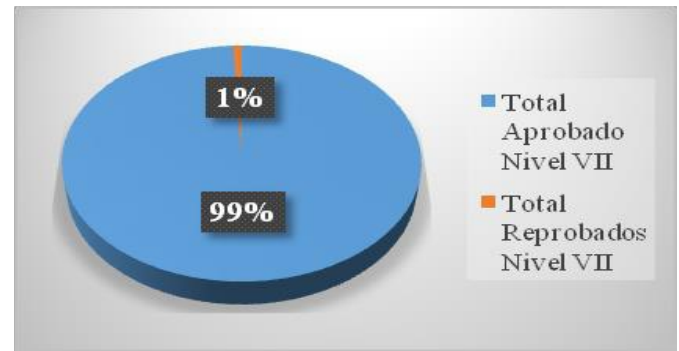

Gráfico 13. Total de estudiantes aprobados vs total de estudiantes reprobados del Nivel VII, septiembre 2013 - julio 2015. 


\section{Análisis e interpretación}

Del 100\% de los estudiantes que cursaron el séptimo nivel de inglés en los diferentes periodos académicos, el 99\% aprobaron, mientras que el 1\% reprobaron. Es decir, la gran mayoría de los estudiantes aprobaron el nivel de inglés VII en los diferentes periodos académicos.

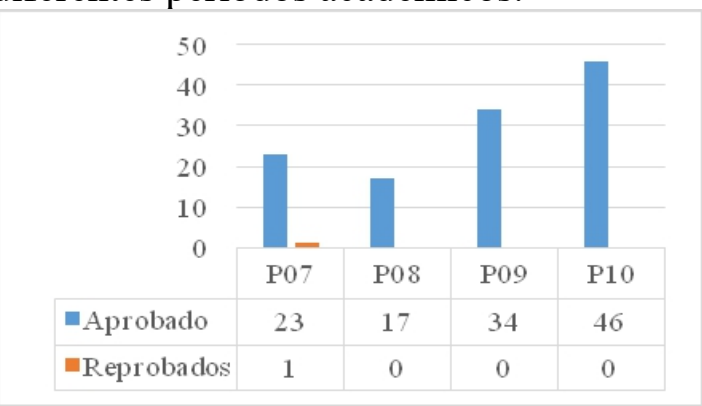

Gráfico 14. Total de estudiantes aprobados vs total de estudiantes reprobados del Nivel VII, septiembre 2013 - julio 2015.

\section{Análisis e interpretación}

El Nivel VII se dictó en 4 periodos académicos desde septiembre 2013 a julio 2015. En el periodo P10, se registró el mayor número de estudiantes aprobados; mientras que en el periodo P08 se registró el menor número de estudiantes aprobados. En el periodo P07, se registró estudiantes reprobados; mientras que en el periodo P08, P09 y P10 no registró reprobados.

\section{Resultados Nivel VIII}

Tabla 8. Total de estudiantes aprobados y reprobados del Nivel VIII, abril 2013 - julio 2015.

\begin{tabular}{cc}
\hline Estado & Número \\
\hline Estudiantes aprobados & 69 \\
Estudiantes reprobados & 2 \\
Total & 71 \\
\hline
\end{tabular}

Fuente: Actas de calificaciones.

Realizado por: Allauca Marcelo, 2015.

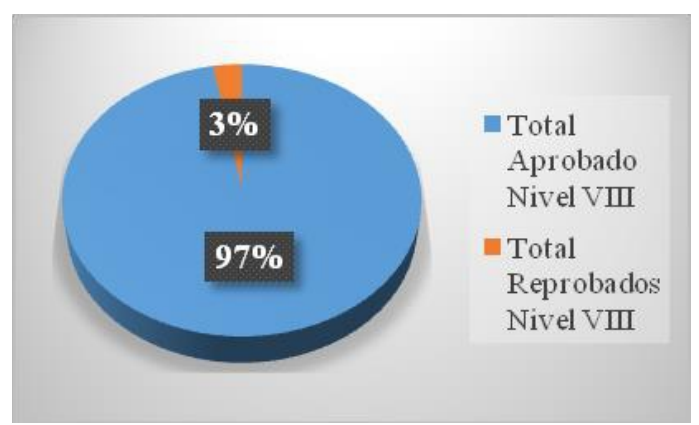

Gráfico 15.Total de estudiantes aprobados vs total de estudiantes reprobados del Nivel VIII, abril 2013 - julio 2015. 


\section{Análisis e interpretación}

Del $100 \%$ de los estudiantes que cursaron el octavo nivel de inglés en los diferentes periodos académicos, el 97\% aprobaron, mientras que el 3\% reprobaron. Es decir, la gran mayoría de los estudiantes aprobaron el nivel de inglés VIII en los diferentes periodos académicos

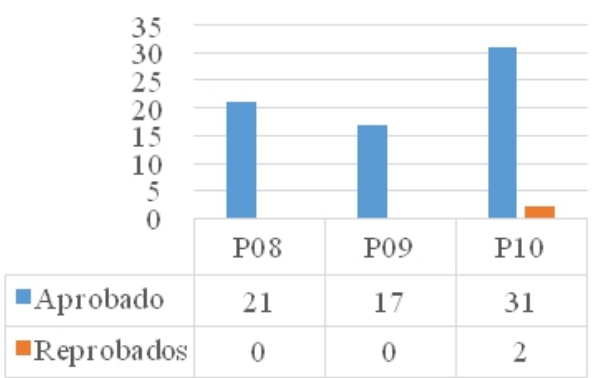

Gráfico 16. Total de estudiantes aprobados vs total de estudiantes reprobados del Nivel VIII, abril 2013 - julio 2015.

\section{Análisis e interpretación}

El nivel VIII se dictó en 3 periodos académicos desde abril 2013 a julio 2015. En el periodo P10 se registró el mayor número de estudiantes aprobados; mientras que en el periodo P08 y P09 no se registró reprobados.

\section{Resultados totales de todos los niveles}

Tabla 9. Total de estudiantes aprobados y reprobados del Nivel VIII, abril 2013 - julio 2015.

\begin{tabular}{cc}
\hline Estado & Número \\
\hline Estudiantes aprobados & 2750 \\
Estudiantes reprobados & 506 \\
Total & 3256 \\
\hline
\end{tabular}

Fuente: Actas de calificaciones.

Realizado por: Allauca Marcelo, 2015.

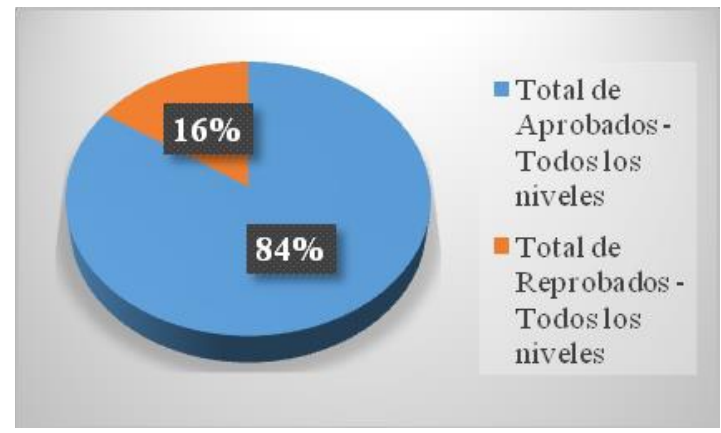

Gráfico 17. Total de estudiantes aprobados vs total de estudiantes reprobados del Nivel VIII, abril 2013 - julio 2015. 


\section{Análisis e interpretación}

Del $100 \%$ de los estudiantes que cursaron algún nivel de inglés en los diferentes periodos académicos, el $84 \%$ aprobaron, mientras que el $16 \%$ reprobaron. Es decir, la mayoría aprobaron un nivel de inglés en los diferentes periodos académicos.

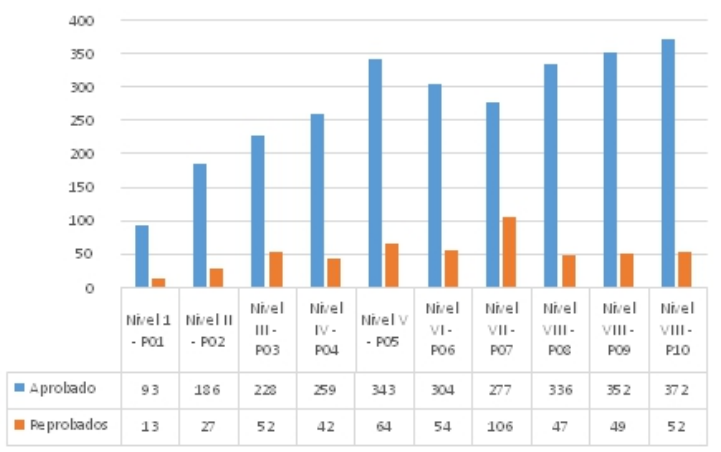

Gráfico 18. Total de estudiantes aprobados vs total de estudiantes reprobados por niveles, abril 2013 - julio 2015.

\section{Análisis e interpretación}

De los niveles que se han dictado en los diferentes niveles comprendidos entre abril 2013 a julio 2015. En el periodo P10 en el Nivel VIII, se registró el mayor número de estudiantes aprobados; mientras que en el periodo $\mathrm{P} 01$ en el primer nivel, se registró el menor número de estudiantes aprobados. En el periodo P07 Nivel VII se registró el mayor número de estudiantes reprobados; mientras que en el Periodo P01 Nivel I se registró el menor número de estudiantes reprobados. Se puede observar que la tendencia de los estudiantes en los niveles superiores fue la aprobación.

Tabla 10. Fechas en que se realizaron las evaluaciones:

\begin{tabular}{cc}
\hline Promoción & Meses \\
\hline P1 & 20 de abril de 2015 \\
P2 & 23 de junio de 2015 \\
P3 & 07 de octubre de 2015 \\
P4 & 05 de diciembre de 2015 \\
\hline
\end{tabular}

Fuente: Actas de calificaciones.

Realizado por: Allauca Marcelo, 2015.

Resultados Promoción 1 (P1)

Tabla 11. Resultados primera promoción

Fecha: 20 de abril de 2015

Primera promoción

\begin{tabular}{|c|c|c|c|c|}
\hline Nivel alcanzado & A2 & B1 & B2 & TOTAL \\
\hline Número de estudiantes & 7 & 13 & 1 & 21 \\
\hline
\end{tabular}

Fuente: Resultados Cambridge.

Realizadopor: Allauca Marcelo, 2015. 


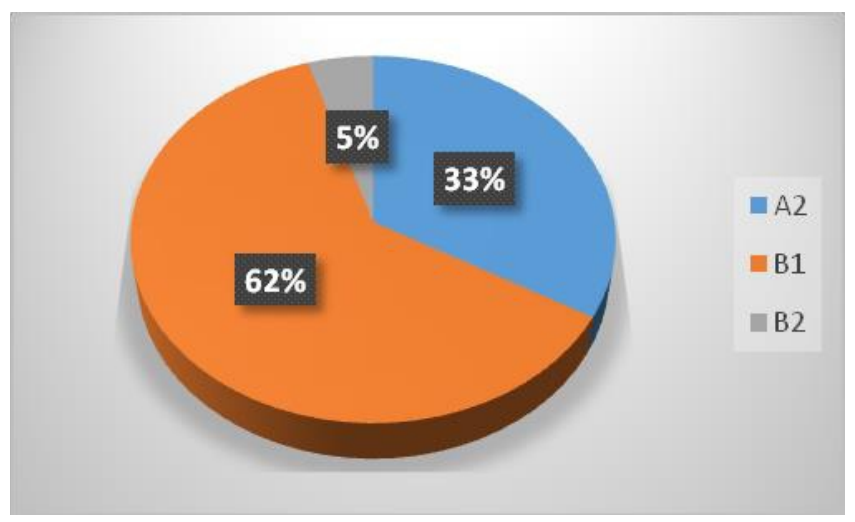

Gráfico 19. Resultados primera promoción.

\section{Análisis e interpretación}

Del $100 \%$ de los estudiantes que rindieron el examen para obtener la certificación internacional en la primera promoción, el 62\% obtuvieron la certificación Nivel B1, el 33\% obtuvieron la certificación Nivel A2; mientras que el 5\% obtuvieron la certificación B2. La mayoría obtuvieron la certificación B1 y B2.

\section{Resultados Promoción 2 (P2)}

Tabla 12. Resultados segunda promoción

\begin{tabular}{|c|c|c|c|c|}
\hline \multicolumn{5}{|c|}{ Fecha: 23 de junio de 2015} \\
\hline Segunda promoción \\
\hline Nivel alcanzado & A2 & B1 & B2 & TOTAL \\
\hline Número de estudiantes & 7 & 9 & 5 & 21 \\
\hline
\end{tabular}

Fuente: Resultados Cambridge.

Realizado por: Allauca Marcelo, 2015.

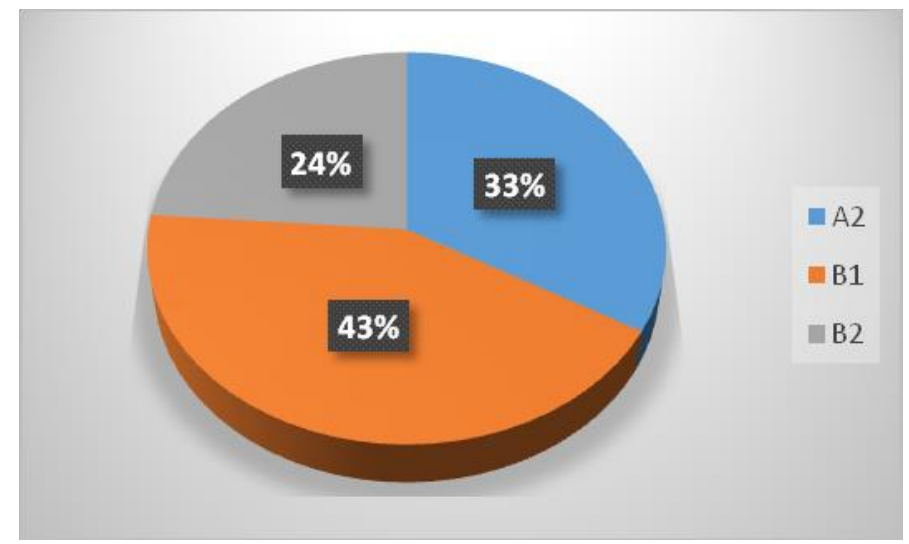

Gráfico 20. Resultados segunda promoción. 


\section{Análisis e interpretación}

Del $100 \%$ de los estudiantes que rindieron el examen para obtener la certificación internacional en la segunda promoción, el 43\% obtuvieron la certificación Nivel B1, el 33\% obtuvieron la certificación Nivel A2; mientras que el $24 \%$ obtuvieron la certificación B2. La mayoría obtuvieron la certificación B1 y B2.

Resultados Promoción 3 (P3)

Tabla 13. Resultados tercera promoción

\begin{tabular}{|c|c|c|c|c|}
\hline \multicolumn{5}{|c|}{ Fecha: 07 de octubre de 2015} \\
\hline \multicolumn{5}{|c|}{ Tercera promoción } \\
\hline Nivel alcanzado & A2 & B1 & B2 & TOTAL \\
\hline Número de estudiantes & 18 & 1 & 16 & 35 \\
\hline
\end{tabular}

Fuente: Resultados Cambridge.

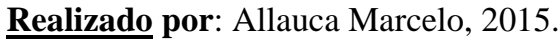

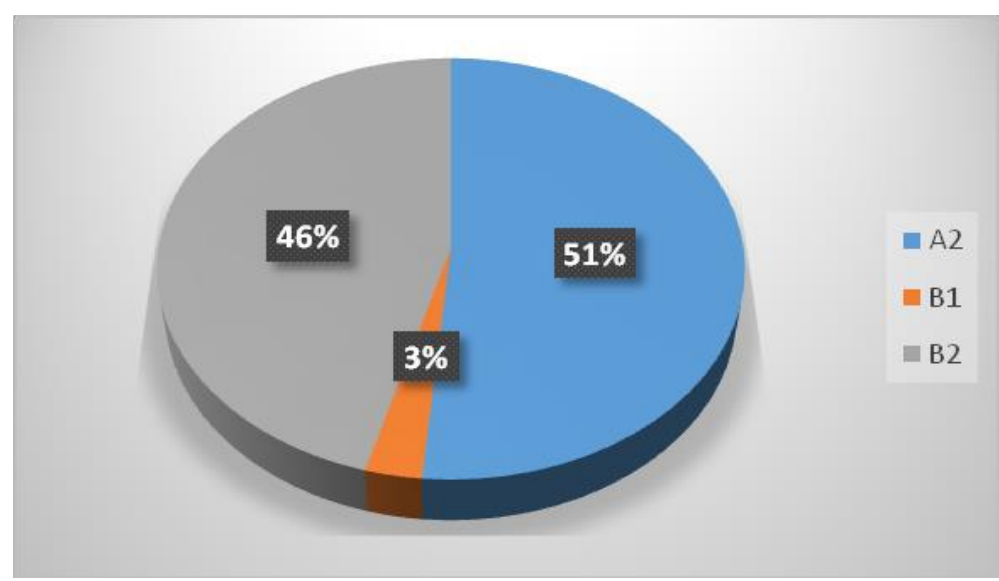

Gráfico 21. Resultados tercera promoción.

\section{Análisis e interpretación}

Del $100 \%$ de los estudiantes que rindieron el examen para obtener la certificación internacional en la tercera promoción, el $46 \%$ obtuvieron la certificación Nivel B2, el 51\% obtuvieron la certificación Nivel A2; mientras que el 3\% obtuvieron la certificación B1. La mayoría obtuvieron la certificación A2.

\section{Resultados Promoción 4 (P4)}

Tabla 14. Resultados cuarta promoción

\begin{tabular}{|c|c|c|c|c|}
\hline \multicolumn{5}{|c|}{ Fecha: 07 de octubre de 2015} \\
\hline \multicolumn{5}{|c|}{ Cuarta promoción } \\
\hline Nivel alcanzado & A2 & B1 & B2 & TOTAL \\
\hline Número de estudiantes & 12 & 33 & 0 & 45 \\
\hline
\end{tabular}

Fuente: Resultados Cambridge.

Realizado por: Allauca Marcelo, 2015. 


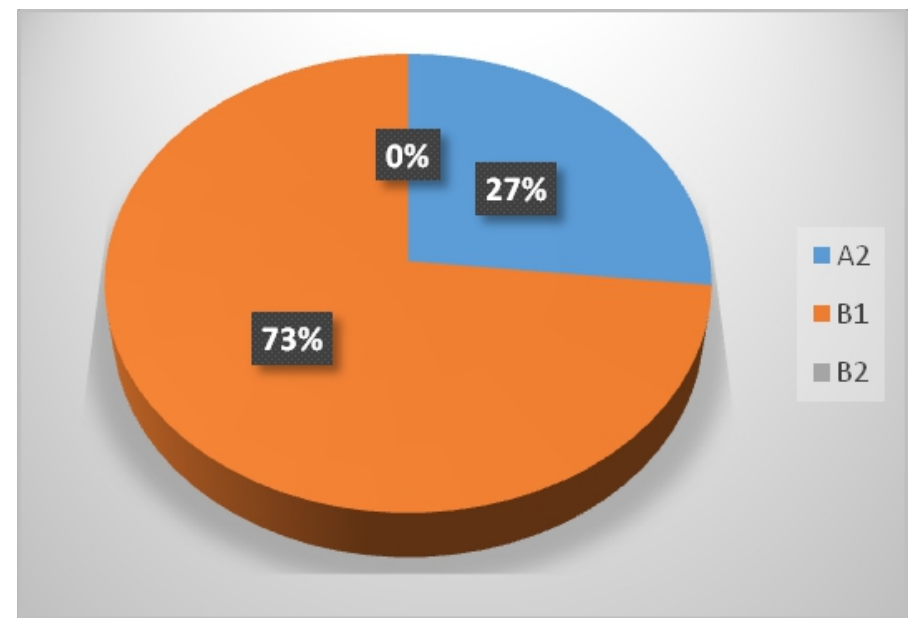

Gráfico 22. Resultados cuarta promoción

\section{Análisis e interpretación}

Del $100 \%$ de los estudiantes que rindieron el examen para obtener la certificación internacional en la cuarta promoción, el 73\% obtuvieron la certificación nivel B1, el 27\% obtuvieron la certificación nivel A2; mientras que no se obtuvieron la certificación B2. La mayoría obtuvieron la certificación B1.

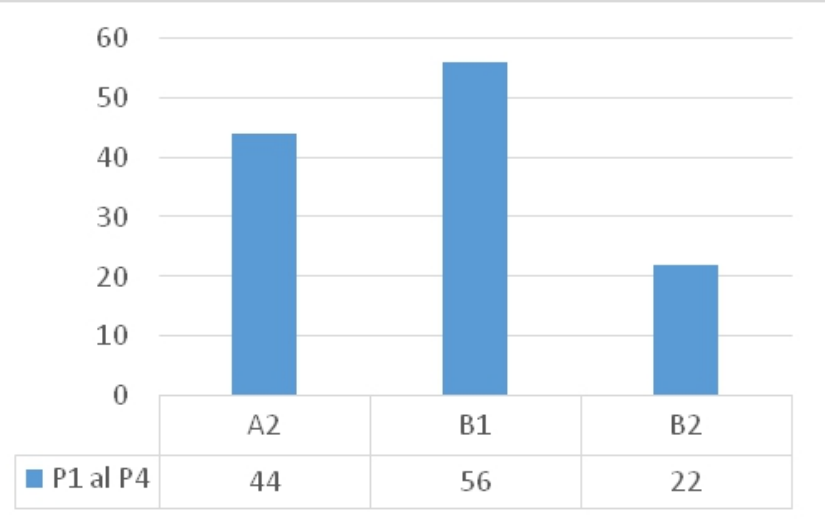

Gráfico 23. Total de estudiantes por niveles de inglés alcanzados.

\section{Análisis e interpretación}

Se puede observar que la certificación B1 representa la mayoría en todas las promociones, seguidas de la certificación A2 y B2. El número de estudiantes que obtuvieron la certificación B2 es bastante considerable desde el punto de vista que el nivel más alto promocionado con la preparación en el Centro de Idiomas. 


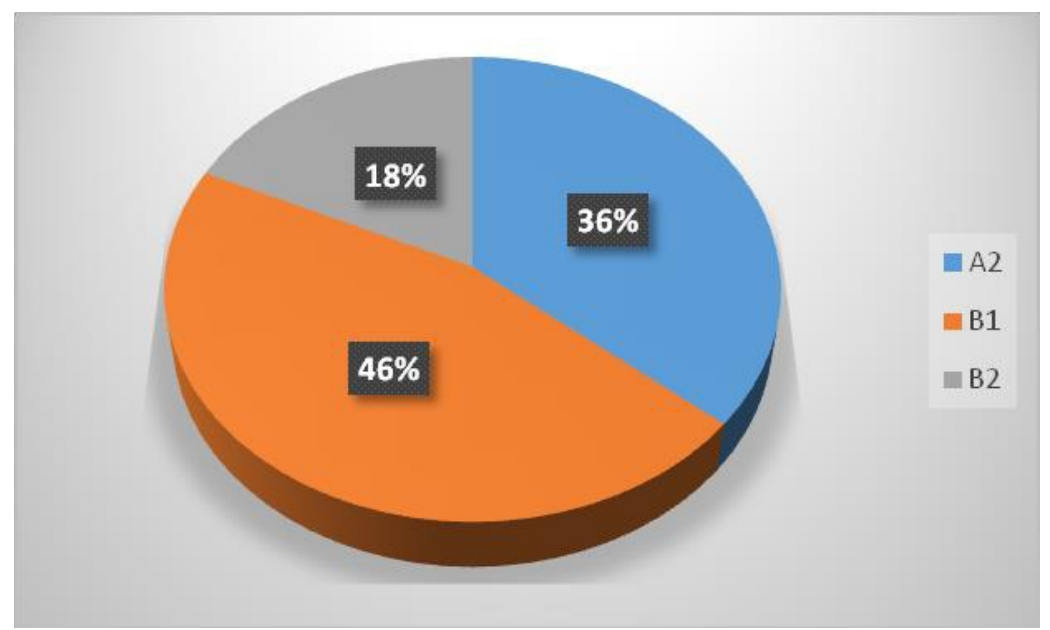

Gráfico 24. Total de estudiantes por niveles de inglés alcanzados.

\section{Análisis e interpretación}

Del $100 \%$ de los estudiantes que rindieron el examen para obtener la certificación internacional en todas las promociones, el 46\% obtuvieron la certificación nivel B1, el 36\% obtuvieron la certificación nivel A2; mientras que el 18\% obtuvieron la certificación B2.

Si sumamos el número de estudiantes que aprobaron el nivel B1 y B2, representan el 64\% de estudiantes que superan al porcentaje del nivel A2.

\section{Conclusiones}

La implementación del proyecto para fortalecer la Modalidad Extensión en el Centro de Idiomas de la Escuela Superior Politécnica de Chimborazo, permitió contar con un centro de preparación avalado por la Certificación de Cambridge para que los estudiantes tengan la posibilidad de prepararse y ser evaluados internamente en las destrezas del inglés durante 720 horas presenciales, previo a la certificación.

El micro currículo y el texto guía que utilizaron los docentes en la preparación de los estudiantes permitió que la preparación en las diferentes destrezas del aprendizaje del inglés sea adecuada y acorde, previa la evaluación de la certificación internacional.

Los resultados de las evaluaciones realizadas a los estudiantes por parte de los representantes de ESOL Cambridge y que se prepararon en la Modalidad Extensión del Centro de Idiomas de la Escuela Superior Politécnica de Chimborazo, permitieron conocer que la mayoría (64\%) de los estudiantes obtuvieron las certificaciones B1 y B2, lo que indica que el objetivo del proyecto fue cumplido satisfactoriamente. 


\section{References:}

1. Alderson Charles, J. (2007). The CEFR and the Need for More Research. The Modern Language Journal, 91/ 4, p 659-663.

2. Cizek Gregory, J., Michael, B., \& Bunch, M.B. (2007). Standard Setting. A guide to Establishing and Evaluating Performance Standards on Tests. Thousand Oaks, London, New Dehli: Sage Publications.

3. Council of Europe (2001). Common European Framework of Reference for Languages: Learning, teaching, assessment. Cambridge: Cambridge University Press.

4. Ecuador Inmediato. (2012, julio 16). Ecuador tiene falencias en enseñanza del Inglés promedio en escuelas y colegios es de 13 sobre 20. Recuperado 3 de marzo de 2016, a partir de http://www.ecuadorinmediato.com/index.php?module=Noticias\&fun c $=$ news_user_view\&id $=177467$

5. El Comercio, M. de E. E. (2014, marzo 26). El déficit de profesores de inglés es un problema que viene desde 1950. Recuperado 3 de marzo de 2016, a partir de http://www.elcomercio.com/tendencias/deficit-deprofesores-de-ingles.html

6. Kaftandjieva Felianka (2004). Standard setting. In Reference Supplement B to the Preliminary Pilot version of the Manual for Relating Language examinations to the Common European Framework of Reference for Languages: learning, teaching, assessment.Strasbourg, Council of Europe.

7. Moe Eli (2008). Standard setting of A2 and B1 items. Standard setting study 1/2008, Norsk språktest, University of Bergen / Folkeuniversitetet, Norway. http://fu.no/default.asp?avd=231\&nyh=6702. 